\title{
Geometrisierung 3-dimensionaler Mannigfaltigkeiten und Ricci-Fluß: Zu Perelmans Beweis der Vermutungen von Poincaré und Thurston \\ von Bernhard Leeb
}

Das Studium qualitativer globaler Eigenschaften von Mannigfaltigkeiten motiviert viele wichtige Entwicklungen in der Topologie. Man möchte Mannigfaltigkeiten bis auf Homöomorphie unterscheiden, d. h. feststellen, ob sie sich durch bijektive (in beide Richtungen) stetige Abbildungen ineinander überführen lassen, anschaulich gesprochen, ob man sie als elastische Objekte aufgefaßt aufeinanderlegen kann, ohne sie zu zerreißen. Besonders interessant sind geschlossene, d. h. kompakte Mannigfaltigkeiten ohne Rand.

\section{Die Poincaré-Vermutung}

In Dimension 2 ist die Situation sehr übersichtlich: Orientierbare geschlossene Flächen werden durch eine einzige numerische Invariante (homologischer Natur) klassifiziert, ihr Geschlecht. Die Gesamtheit aller 3-dimensionalen Mannigfaltigkeiten hingegen ist ungleich komplexer. Dies wird schon klar, wenn man nur an die Vielgestalt von Knoten in der 3dimensionalen Sphäre $S^{3}$ denkt: Die Komplemente offener Tubenumgebungen um Knoten sind kompakte 3-dimensionale Mannigfaltigkeiten mit einem Randtorus, und man kann je zwei von ihnen entlang ihrer Randtori zu einer geschlossenen Mannigfaltigkeit verkleben.

Poincaré schlug eine Charakterisierung von $S^{3}$ vor, gewissermaßen der „einfachsten“ geschlossenen 3Mannigfaltigkeit. Anschaulich läßt sie sich folgendermaßen aussprechen. Dabei ist mit einer Schleife ein geschlossener Weg gemeint, der sich selbst durchdringen darf.

PV (1904). Jede geschlossene 3-Mannigfaltigkeit, in welcher sich alle Schleifen stetig auf einen Punkt zusammenziehen lassen, ist homöomorph zu $S^{3}$.

Als Frage formuliert steht die PV am Ende einer Serie von Arbeiten Henri Poincarés zur Analysis Situs (1895-1904). Er begründet dort die Algebraische Topologie, d. h. die Entwicklung algebraischer Invarianten zur topologischen Unterscheidung von Mannigfaltigkeiten und allgemeineren Räumen. Eine typische algebraisch-topologische Invariante einer Mannigfaltigkeit $M$, eingeführt bereits von Poincaré, ist ihre Fundamentalgruppe $\pi_{1}(M)$. Man faßt alle Schleifen in $M$ mit fest gewähltem Fußpunkt zu Äquivalenzklassen modulo stetiger Deformation („Homotopie“) zusammen. Es ergibt sich so ein diskretes Objekt mit einer natürlichen algebraischen Struktur, nämlich einer Gruppenstruktur gegeben durch das Hinterein- anderdurchlaufen von Schleifen. Die PV behauptet, daß $S^{3}$ durch die Trivialität ihrer Fundamentalgruppe charakterisiert ist.

Die zunächst gefundenen algebraisch-topologischen Invarianten wie Homologie und Fundamentalgruppe können nur bis auf die gröbere Relation der Homotopie-Äquivalenz unterscheiden, d.h. sie sind insensitiv für die Unterscheidung von Räumen, die zwar nicht homöomorph sind, sich aber in einem gewissen allgemeineren Sinne ineinander deformieren lassen. In Dimension 3 reicht die Trivialität der Fundamentalgruppe bereits aus, damit eine geschlossene Mannigfaltigkeit eine Homotopie-Sphäre, d.h. homotopie-äquivalent zur Standard-Sphäre $S^{3}$ ist. Konzeptioneller formuliert lautet die PV demnach:

$\mathbf{P V}^{\mathbf{3}}$. Jede 3-dimensionale Homotopie-Sphäre ist homöomorph zu $S^{3}$.

Die analoge Frage $\mathrm{PV}^{\mathrm{m}}$ ist bereits seit etwa 25 Jahren in allen Dimensionen $m \neq 3$ gelöst: Zunächst in „höherer" Dimension $\geq 5$ durch eine spektakuläre Anwendung der Morse-Theorie (Smale 1962, FieldsMedaille 1966) und 20 Jahre später von Freedman in Dimension 4 als Konsequenz seiner Klassifikation topologischer 4-Mannigfaltigkeiten mit trivialer Fundamentalgruppe (1982, Fields-Medaille 1986).

\section{Geometrisierung}

Das von Thurston in den 70er Jahren aufgestellte Geometrisierungs-Programm veränderte die Landschaft in der 3-dimensionalen Topologie nachhaltig. Es stellte eine enge Verbindung zur Differentialgeometrie her, insbesondere zur hyperbolischen Geometrie und der Theorie Kleinscher Gruppen.

Die allgemeine Idee ist folgende: Eine Mannigfaltigkeit zu geometrisieren bedeutet, sie mit einer „schönen" geometrischen Struktur zu versehen, in unserem Fall mit einer Riemannschen Metrik. Das ursprünglich „wabbelige" topologische Objekt wird so in eine starre geometrische Form gegossen. Diese soll möglichst günstig sein, um die topologischen Eigenschaften besser sichtbar zu machen.

In Dimension 2 leistet dies der klassische Uniformisierungssatz für Flächen in abschließender Weise. Es war Thurstons fundamentale und überraschende Einsicht, daß eine ähnlich starke Uniformisierung auch in Dimension 3 noch möglich sein sollte. Beginnen wir damit, die PV aus Sicht der Geometrisierung zu formulieren: 
PV'geom. Jede geschlossene 3-Mannigfaltigkeit mit trivialer Fundamentalgruppe trägt eine sphärische Struktur.

Unter einer sphärischen Struktur auf einer 3dimensionalen Mannigfaltigkeit verstehen wir eine (Riemannsche) Metrik, vermittels derer sie lokal isometrisch zur 3-dimensionalen Einheitssphäre wird. Im Kontrast zu den "flexiblen" topologischen Mannigfaltigkeiten kann man sich geometrische Mannigfaltigkeiten als „starre“ Objekte vorstellen. Erstere sind aus Stückchen euklidischen Raumes ,zusammengenäht", wobei man beim Vernähen beliebig dehnen darf, solange man nicht zerreißt. Letztere aus Stückchen eines metrischen Modellraumes und es muß isometrisch vernäht werden.

Die Implikation $\mathrm{PV}^{\text {geom }} \Rightarrow \mathrm{PV}$ ist elementar und folgt aus der Eindeutigkeit der Modellräume konstanter (Schnitt)Krümmung: Die Einheitssphäre ist bis auf Isometrie die einzige geschlossene sphärische Mannigfaltigkeit mit trivialer Fundamentalgruppe.

Die PVgeom geht in einer allgemeineren Vermutung Thurstons auf, die genau beschreibt, wann sphärische Strukturen existieren:

Elliptisierungs-Vermutung (TEV). Jede geschlossene 3-Mannigfaltigkeit mit endlicher Fundamentalgruppe trägt eine sphärische Struktur.

Motiviert durch überraschende Konstruktionen hyperbolischer Strukturen u. a. auf vielen Komplementen von Knoten in $S^{3}$ und verwandten geschlossenen 3-Mannigfaltigkeiten (z.B. durch hyperbolische Dehn-Chirurgie) hatte Thurston auch hier eine präzise Vermutung formuliert:

Hyperbolisierungs-Vermutung (THV). Jede irreduzible geschlossene 3-Mannigfaltigkeit, deren Fundamentalgruppe unendlich ist und keine Untergruppe isomorph zu $\mathbb{Z}^{2}$ besitzt, trägt eine hyperbolische Struktur.

Man kann beide Vermutungen so paraphrasieren, daß auch in Dimension 3 sphärische bzw. hyperbolische Strukturen existieren, sobald die offensichtlichen algebraisch-topologischen Obstruktionen nicht vorliegen. Im hyperbolischen Fall kommt außerdem die Forderung der Irreduzibilität hinzu, weil man nichttriviale zusammenhängende Summen ${ }^{1}$ ausschließen muß. Sie besagt, daß jede eingebettete 2 -Sphäre einen eingebetteten 3-Ball berandet.

Die sich in der THV ausdrückende Flexibilität der hyperbolischen Geometrie lag keinesfalls auf der Hand. Z. B. besagt Mostow-Starrheit, daß geschlossene Mannigfaltigkeiten der Dimension $\geq 3$ höchstens eine hyperbolische Struktur zulassen, d. h. eine solche nur trivial deformiert werden kann, während auf Flächen höheren Geschlechts ganze Moduli existieren. Thurston untermauerte seine Vermutung mit umfangreicher Evidenz, indem er sie im Haken-Fall bewies. Dabei behandelte er auch kompakte Mannigfaltigkeiten mit Rand, womit u. a. alle Knotenkomplemente in $S^{3}$ erfaßt sind.

TEV und THV waren die beiden vor Perelman weit offenen Teile des Geometrisierungs-Programms, das sich auf die Gesamtheit aller (kompakten) 3Mannigfaltigkeiten erstreckt. Es sagt aus, daß man auch in Dimension 3 noch geometrisch uniformisieren kann, jedoch erst, nachdem man die Mannigfaltigkeiten einem kanonischen topologischen Zerlegungsprozeß unterworfen hat.

Geometrisierungs-Vermutung (TGV). Jede geschlossene 3-Mannigfaltigkeit läßt sich auf kanonische Weise topologisch in geometrische Komponenten zerlegen.

Was damit genauer gemeint ist, können wir hier nur grob umreißen.

Die sphärische, euklidische und hyperbolische Geometrie, also die isotropen Riemannschen Strukturen, reichen zur Geometrisierung in Dimension 3 nicht mehr aus. Es stellt sich als natürlich heraus, allgemeiner homogene Modellgeometrien zuzulassen. Acht Typen solcher Geometrien treten auf: Zu den Modellräumen konstanter Krümmung $\left(S^{3}, R^{3}, \mathbb{H}^{3}\right)$ kommen die Produktgeometrien $\left(S^{2} \times \mathbb{R}, H^{2} \times \mathbb{R}\right)$ hinzu sowie drei ,getwistete" Geometrien $(N i l, P \widetilde{S L(2, \mathbb{R})}, S o l)$, die wir für unsere weitere Diskussion aber nicht genauer kennen müssen. Eine schöne Darstellung findet man in $[\mathrm{Sc}]$.

Die topologische Zerlegung erfolgt durch Zerschneiden entlang von Flächen in zwei Etappen. Der erste Schritt ist die Primzerlegung (Kneser 1929, Milnor 1962) entlang separierender Sphären. Die (eindeutigen) „Primfaktoren“ erhält man durch Einfüllen von Bällen in die beim Schneiden entstandenen Randsphären. Im zweiten Schritt, der TorusZerlegung (Jaco-Shalen und Johannson, Ende 70er), zerschneidet man die irreduziblen Primkomponenten mit unendlicher Fundamentalgruppe entlang von Tori weiter in so genannte Seifert- und atoroidale Komponenten, die ebenfalls eindeutig sind. Jeder der beiden Zerlegungsschritte kann trivial sein. Die TEV betrifft die Primkomponenten mit endlicher Fundamentalgruppe und die THV die atoroidalen Komponenten in der Torus-Zerlegung. Die Geometrisierung der restlichen Komponenten ist unproblematisch.

1 Die zusammenhängende Summe $M_{1} \sharp M_{2}$ zweier Mannigfaltigkeiten $M_{1}$ und $M_{2}$ gleicher Dimension bildet man, indem man aus jeder von ihnen einen offenen Ball entfernt und sie dann entlang der Randsphären verklebt. 


\section{Krümmung}

Um den Ricci-Fluß genauer diskutieren zu können, gehen wir kurz auf die Hierarchie der geläufigsten Krümmungsgrößen in der Differentialgeometrie ein Schnitt-, Ricci- und Skalarkrümmung - und versuchen, eine grobe anschauliche Vorstellung zu vermitteln.

Eine Riemannsche Metrik $g$ auf einer Mannigfaltigkeit $M$ ist eine glatt vom Fußpunkt $p$ abhängige Familie von Skalarprodukten auf den Tangentialräumen $T_{p} M$. Sie spezifiziert also die Längen und Winkel von Tangentialvektoren. Krümmung ist die fundamentale differentialgeometrische Invariante, um Riemannsche Metriken bis auf Isometrie unterscheiden zu können. Die gesamte Information über die Krümmung einer Riemannschen Metrik steckt in ihrem Riemannschen Krümmungstensor $R$, den man rein formal als Tensorfeld vom Typ $(0,4)$ definieren kann, d. h. als glatt vom Fußpunkt abhängige Familie von Quadrilinearformen auf den Tangentialräumen, die außerdem gewisse Symmetrien aufweisen. Der Krümmungstensor in einem festen Punkt hängt von der Metrik und deren Ableitungen bis zur 2. Ordnung ab.

In Dimension 2, auf einer Fläche, reduziert sich $R$ zu einer skalaren Funktion, der Gauß-Krümmung $K$. Man kann sie im Punkt $p$ am infinitesimalen Wachstumsverhalten der Umfänge $L(p, r)$ der Kreise vom Radius $r$ ablesen:

$$
K(p)=-\frac{1}{2 \pi} L^{\prime \prime \prime}(p, 0)
$$

In beliebiger Dimension ist jedem 2-dimensionalen Unterraum e $\subset T_{p} M$ eines Tangentialraums seine Schnittkrümmung $\sec (e)$ zugeordnet, die man als Gauß-Krümmung eines Flächenstücks durch $p$ bestehend aus Geodätischen tangential an $e$ interpretieren kann. Die Information in den Schnittkrümmungen und die im Krümmungstensor sind äquivalent.

Diese Information kann man in zwei Schritten ausdünnen: Die Kontraktion von $R$ liefert den RicciTensor Ric. Er ist ebenso wie $g$ ein symmetrisches $(0,2)$-Tensorfeld, d. h. eine Familie symmetrischer Bilinearformen. Für einen Einheitstangentialvektor $v \in$ $T_{p} M$ ist die Ricci-Krümmung $\operatorname{Ric}(v, v)$ bis auf einen Normierungsfaktor das Mittel der Schnittkrümmungen $\sec (e)$ aller Ebenen $e \subset T_{p} M$ tangential an $v$. Eine weitere Kontraktion, jetzt von Ric, liefert die Skalarkrümmung scal, eine Funktion auf $M$. Bis auf einen Faktor ist $\operatorname{scal}(p)$ das Mittel über die Schnittkrümmungen aller Ebenen in $T_{p} M$. In Dimension 2 sind Gauß- und Skalarkrümmung proportional.

\section{Der Ricci-Fluß}

Wie findet man schöne Metriken auf einer gegebenen Mannigfaltigkeit?

Die Überlegungen verlagern sich nun in die Geometrische Analysis, ein Wechselspiel aus Globaler Differentialgeometrie und Nichtlinearen Partiellen Differentialgleichungen. Topologische Betrachtungen treten in den Hintergrund, geben aber die große Linie vor und motivieren viele der konkreten Schritte.

Ein natürlicher analytischer Ansatz ist nämlich, von einer (beliebigen) Startmetrik $g_{0}$ ausgehend zu versuchen, diese zu Metriken mit besseren geometrischen Eigenschaften zu deformieren und schließlich zu optimieren. Mit beliebigen Startmetriken muß man zurande kommen, weil man auf potentiellen Gegenkandidaten zu den Vermutungen a priori keine Metriken mit besonderen Eigenschaften herstellen kann.

Hamilton suchte zu diesem Zweck nach einem „Wärmefluß für Riemannsche Metriken", also einer sinnvollen Partiellen Differentialgleichung (PDE) der Bauart,$\dot{g}=\Delta g^{\prime}$. Er hoffte, ein solcher Prozeß könne beliebige Startmetriken homogenisieren, indem er ihre Krümmung über die Mannigfaltigkeit „verteilt“. Wörtlich genommen, verschwindet die rechte Seite $\Delta g$ allerdings für den durch $g$ selbst bestimmten Laplace-Operator. Hamilton wollte sie deshalb durch einen Ausdruck ersetzen, der aus (bis zu) zweifachen Ableitungen der Metrik aufgebaut ist. Krümmungsgrößen sind von dieser Art! Die Ricci-Krümmung bietet sich besonders an, weil sie ein Tensor vom Typ der Riemannschen Metrik ist. Außerdem enthält Ric in Dimension 3 noch die gesamte Information von $R$. Von dieser Heuristik geleitet schlug Hamilton 1982 seine Ricci-Fluß-Gleichung vor:

$$
\dot{g}=-2 R i c_{g}
$$

Es handelt sich um eine schwach parabolische PDE zweiter Ordnung. Das negative Vorzeichen stellt sicher, daß sie einem vorwärtsgerichteten Wärmefluß entspricht und nicht einem rückwärts ablaufenden, einer „Wärmeakkumulation“, von der man natürlich weder Glättungsverhalten noch Kurzzeitexistenz erwarten kann.

Daß die Ricci-Fluß-Gleichung nur schwach parabolisch ist, liegt an ihrer Invarianz unter der Diffeomorphismengruppe. Die Kurzzeit-Existenz gilt trotzdem auf jeder geschlossenen Mannigfaltigkeit $M^{m}$. Zu jeder Startmetrik $g_{0}$ gibt es eine eindeutige maximale Lösung $\left(g_{t}\right)$ für $0 \leq t<T$ mit Entartungszeit $T \in(0,+\infty]$. Wir werden einen solchen Ricci-Fluß als Raum-Zeit $M \times[0, T)$ auffassen und von dem mit der Metrik $g_{t}$ versehenen raumartigen ,Slice“ $M \times\{t\}$ bzw. $\left(M, g_{t}\right)$ sprechen. 


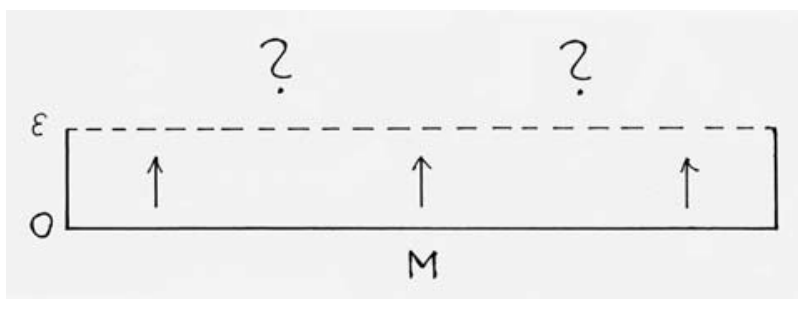

Abbildung 1. Kurzzeit-Existenz

Homogenität wird vom Ricci-Fluß erhalten, und für homogene Metriken wird die PDE zur ODE. Man erhält so einen sehr begrenzten Vorrat an expliziten Lösungen, die eine gewisse grobe Vorstellung vom qualitativen Verhalten des Ricci-Flusses im allgemeinen Fall vermitteln. Im Fall $s e c \equiv$ const gilt: Flache Mannigfaltigkeiten sind statisch, sphärische kollabieren in endlicher Zeit mit Skalierungsfaktor $(1-4 t)^{\frac{1}{2}}$ und hyperbolische expandieren mit Skalierungsfaktor $(1+4 t)^{\frac{1}{2}}$ für alle Zeiten.

Die modulo Reskalierung stationären Metriken sind genau die Einstein-Metriken, d. h. in Dimension 3 die Metriken mit sec $\equiv$ const. Da es solche Metriken auf einer gegebenen 3-Mannigfaltigkeit im allg. nicht gibt, sind Degenerationen unvermeidlich. Mit großem Optimismus und starker Vision kann man auf gute geometrisch-analytische Kontrolle im allgemeinen Fall hoffen und den folgenden Bezug zu Thurstons Programm herstellen, vgl. [H2, H3]:

Hamiltons Programm (HP). In Dimension 3 sind die Degenerationen der Metriken $\left(g_{t}\right)$ unter dem Ricci-Fluß geometrisch gut beschreibbar, und zwar hinreichend präzise, um den Ricci-Fluß mittels einer geeigneten Modifikation (Chirurgie) „durch die Singularitäten hindurch" ad infinitum fortsetzen zu können.

Die Degenerationen und das asymptotische Langzeitverhalten des Ricci-Flusses bringen die topologische Zerlegung von $M$ in geometrische Komponenten zum Vorschein. Genauer: Die zu endlichen Zeiten stattfindenden Degenerationen stehen im Zusammenhang mit der Primzerlegung. Die Torus-Zerlegung wird in den Entartungen zur Zeit $+\infty$ hin sichtbar. (Im allg. sind nicht alle Degenerationen topologisch relevant, sondern es gibt auch topologisch „überflüssige“.)

Daß der Ricci-Fluß eine exzellente Wahl ist und seine Nützlichkeit für die 3-dimensionale Topologie kein bloßes Wunschdenken, belegte Hamilton gleich zu Beginn mit einem beeindruckenden Resultat [H1], das als Grundstein seines Programms gelten kann: Falls Ric $_{g_{0}}>0$, so schrumpft $\left(M^{3}, g_{t}\right)$ in endlicher Zeit T $z u$ einem Punkt. Für $t \nearrow T$ wird $\left(M^{3}, g_{t}\right)$ immer runder und konvergiert modulo Reskalierung, z.B. bei Normierung des Volumens, gegen eine sphärische Metrik. Eine relativ schwache Positivitätsbedingung an die Krümmung führt also zur bestmöglichen. Bis vor kurzem war dieses Resultat das stärkste in Richtung Elliptisierung.

\section{Evolution der Krümmung}

Die Kontrolle der Geometrie der evolvierenden Metriken $g_{t}$ basiert auf der Beobachtung, daß die zeitliche Entwicklung der Krümmung ebenfalls einer parabolischen PDE vom Wärmeleitungstyp gehorcht. Für den vollen Krümmungstensor hat sie die Struktur:

$$
\dot{R}=\Delta R+\text { homogen quadratisch }(R) .
$$

Der Diffusionsterm $\Delta R$ unterstützt die erhoffte Verteilung der Krümmung, während der Reaktionsterm dagegen arbeiten kann.

Man stellt eine generelle Tendenz des Ricci-Flusses fest, die Bildung positiver Krümmung zu fördern sowie positive Krümmungsbedingungen zu erhalten und zu verbessern. Das erste Indiz ist die PDI

$$
\text { scal } \geq \Delta \mathrm{scal}+\frac{2}{m} \cdot \mathrm{scal}^{2}
$$

die sich durch Mittelung aus der Evolution von $R$ ergibt. Eine einfache Anwendung des skalaren Maximumprinzips zeigt, daß das globale Minimum der Skalarkrümmung der Metriken $g_{t}$ im Laufe der Zeit ansteigt:

$$
\min \operatorname{scal}(\cdot, t) \nearrow \quad \text { in } t
$$

Startet man bereits mit einer Metrik positiver Skalarkrümmung, so liefert ein simpler ODE-Vergleich eine obere Schranke an die Lebenszeit $T$.

Auch für die feineren Krümmungsgrößen Ric und sec sind Abschätzungen der zeitlichen Entwicklung möglich. Das entscheidende Werkzeug ist ein von Hamilton entwickeltes Maximumprinzip für Tensoren. Er wendete es zuerst in [H1] an, um zu zeigen, daß sich auf 3-Mannigfaltigkeiten mit Ric $>0$ die Krümmung unter dem Ricci-Fluß immer gleichmäßiger verteilt. Solche Resultate gelten unter geeigneten (wesentlich stärkeren) Positivitätsbedingungen an die Krümmung auch in allen höheren Dimensionen.

Ab jetzt beschränken wir uns auf Dimension 3. Wir werden später der Einfachheit halber auch annehmen, daß unsere geschlossene Mannigfaltigkeit $M^{3}$ orientierbar ist. Um das HP überhaupt in Gang zu bringen, ist entscheidend, daß man Kontrolle über den Ricci-Fluß bekommt, ohne Voraussetzungen an die Krümmung der Startmetrik zu machen. Dies wird durch eine weitere raffinierte Anwendung des tensoriellen Maximumprinzips ermöglicht. Das HamiltonIvey Pinching liefert eine Abschätzung des negativen Anteils der Schnittkrümmungen von der Form

$$
\sec \geq-\phi(\text { scal })
$$


mit einer sublinearen Funktion $\phi: \mathbb{R} \rightarrow[0, \infty)$, d. h. $\lim _{s \rightarrow+\infty} \frac{\phi(s)}{s}=0$, die alleine von den Schranken an die Krümmung der Startmetrik abhängt.

Die Konsequenzen sind erstaunlich: Man findet sich in der ungewohnten Situation wieder, daß die Schnittkrümmungen punktweise von der Skalarkrümmung (ihrem Mittelwert!) kontrolliert werden. Die Degeneration des Ricci-Flusses in endlicher Zeit $T$ ist daher auch gleichbedeutend mit Explosion der Skalarkrümmung,

$$
\max \operatorname{scal}(\cdot, t) \nearrow+\infty \quad \text { für } t \nearrow T .
$$

Das bedeutet i. allg. nicht, daß scal - wie im Fall Ric $>0$ - überall auf $M$ gleichmäßig groß wird. Wo aber die Krümmung explodiert, dominieren die positiven Schnittkrümmungen die negativen, und zwar umso stärker, je höher die Skalarkrümmung wird. Relativ gesehen entwickelt sich also in den Regionen mit $s c a l \simeq+\infty$ fast nichtnegative Schnittkrümmung. Die Bevorzugung positiver Krümmung durch den RicciFluß bestätigt sich hier ein weiteres Mal. (All dies schließt nicht aus, daß der absolute Wert von sec beliebig negativ werden kann, aber eben viel langsamer als scal anwächst.)

\section{Singularitäten-Modelle}

Um lokale Modelle für die Regionen mit scal $\simeq+\infty$ zu beschreiben, in denen sich die Singularitäten bilden, ,zoomt" man entlang einer Folge von Punkten $\left(x_{k}, t_{k}\right)$ aus $M \times[0, T)$ mit $\operatorname{scal}\left(x_{k}, t_{k}\right) \nearrow+\infty$ hinein. Man möchte aus der Folge von Flüssen $\left(g_{t}^{(k)}\right)$, die nach Normalisierung der Skalarkrümmung in den Fußpunkten $\left(x_{k}, t_{k}\right)$ entstehen, einen Limes-Fluß $\left(g_{t}^{(\infty)}\right)$ extrahieren. Solche Limes-Flüsse würden Regionen hinreichend hoher Skalarkrümmung gut approximieren und hätten ganz besondere Eigenschaften: Aufgrund der Reskalierung - die hier in räumlicher und zeitlicher Richtung stattfindet ${ }^{2}$ - existieren die Flüsse $\left(g_{t}^{(k)}\right)$ rückwärts für immer längere Zeiten, und Limes-Flüsse wären Urlösungen (ancient solutions), d. h. für alle negativen Zeiten $-\infty<$ $t \leq 0$ definiert. Das bedeutet, daß man mit den Metriken $\left(g_{t}^{(\infty)}\right)$ als Anfangsbedingungen den rückwärtsgerichteten Ricci-Fluß ad infinitum lösen könnte, diese Metriken also „stark geglättet“ wären. Als Folge des Hamilton-Ivey-Pinchings würden sie außerdem die starke Krümmungsbedingung

$$
\sec _{g_{t}^{(\infty)}} \geq 0
$$

erfüllen. Dies brächte die gut entwickelte Strukturtheorie für Riemannsche Mannigfaltigkeiten und

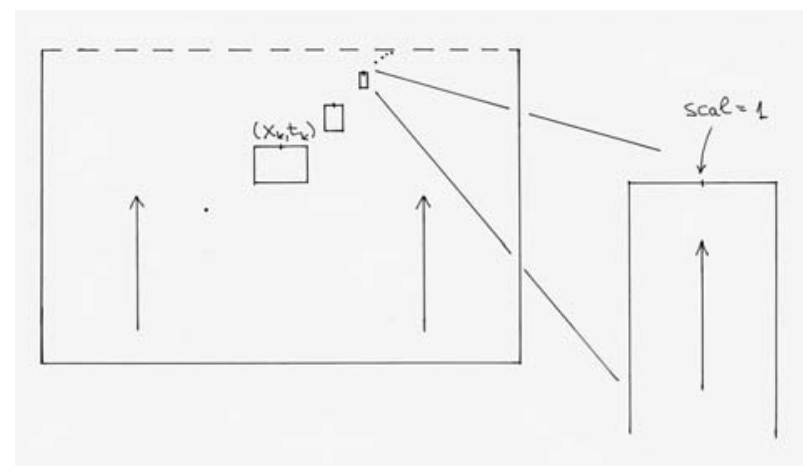

Abbildung 2. Zoom

allgemeinere singuläre (Aleksandrov-)Räume mit $s e c \geq 0$ bei der Analyse der Singularitäten des RicciFlusses zum Tragen. Es sei angemerkt, daß die den Limes-Flüssen zugrundeliegenden Mannigfaltigkeiten im allg. von $M$ verschieden und nicht mehr kompakt wären.

Auf dieser von Hamilton vorgezeichneten Route konnten vor Perelmans Arbeiten [Pe1, Pe2] ernsthafte Hürden nicht überwunden werden und die Entwicklung stagnierte. $\mathrm{Zu}$ den Hauptproblemen gehört die Kontrolle der Krümmung der Metriken $g_{t}^{(k)}$ und das Ausschließen von Kollaps. Denn auch bei bester Krümmungskontrolle kann eine Folge von Riemannschen Mannigfaltigkeiten (oder Ricci-Flüssen) zu einem Limes-Objekt niedrigerer Dimension kollabieren. Ein typisches Beispiel ist die Konvergenz (im Gromov-Hausdorff-Sinne) einer Folge immer dünnerer flacher Zylinder zur Geraden.

Perelman gelang in [Pe1] der Durchbruch zur Realisierung des HP. Dort und in den darauffolgenden Arbeiten [Pe2, Pe3] brachte er zu den bereits vorhandenen analytischen Techniken einen neuen globaldifferentialgeometrischen Aspekt ein, gekennzeichnet durch einzigartiges geometrisches Fingerspitzengefühl. In einigen Gebieten der Geometrie, die hier eine Rolle spielen, hatte er vorher substantielle Beiträge geleistet. Etwa in der Strukturtheorie von Mannigfaltigkeiten mit $s e c \geq 0$, wo er die Seelen-Vermutung von Cheeger und Gromoll bewiesen hatte (1994), oder in der Theorie der Aleksandrov-Räume mit unterer Krümmungsschranke, die er maßgeblich mitentwickelte.

Perelman zeigte in [Pe1], daß der Ricci-Fluß nach gewisser Laufzeit eine Nichtkollabiertheit entwickelt. Grob gesprochen heißt dies, daß Bälle auf einer der Skalarkrümmung entsprechenden Skala ein nicht zu kleines Volumen haben. Die Rate des Nichtkollaps hängt von Startmetrik $g_{0}$ und Laufzeit ab. Er konnte die erwähnten Limes-Flüsse extrahieren und so die

2 Um wieder Lösungen der Ricci-Fluß-Gleichung zu erhalten, muß gemäß ihrer Struktur der zeitliche Skalierungsfaktor das Quadrat des räumlichen sein. Man spricht von parabolischer Reskalierung. 
Urlösungen als lokale Modelle für die Bereiche mit $s c a l \simeq+\infty$ etablieren.

Die Topologie der Urlösungen ergibt sich im kompakten Fall bereits aus [H1] und im nichtkompakten Fall aus der Strukturtheorie (dem „Seelensatz“) für nichtkompakte Mannigfaltigkeiten mit $s e c \geq 0$ (Cheeger und Gromoll, 1972). In [Pe1, Pe2] beschrieb Perelman auch ihre Geometrie und gab eine Klassifikation an. Hier nur ein kurzes Wort zum nichtkompakten Fall: Mannigfaltigkeiten mit sec $\geq 0$ öffnen sich immer höchstens so schnell wie der euklidische Raum. Ein typisches Beispiel ist ein Rotationsparaboloid. Die räumlichen Querschnitte der Urlösungen öffnen sich sogar so langsam, daß sie asymptotisch zylindrisch sind. Außerhalb eines Bereichs kontrollierbarer Ausdehnung sind sie halsartig, d. h. beliebig gut approximierbar durch runde Zylinder. Dies liefert die nötige geometrische Kontrolle, um eine präzise Chirurgie ansetzen zu können.

Durch Zusammensetzen der lokalen Information erhält man ein gutes Bild, wie sich die globale Geometrie der Metriken $g_{t}$ für $t \nearrow T$ entwickelt, vgl. [Pe2]. Es gibt eine echte offene Teilmenge $\Omega \subset M$, auf welcher die Krümmung punktweise beschränkt bleibt und die Metriken glatt gegen eine Limes-Metrik konvergieren,

$$
g_{t} \stackrel{\mathcal{C}^{\infty}}{\longrightarrow} g_{T} \quad \text { auf } \Omega .
$$

$\mathrm{Zu}$ den Enden von $\Omega$ hin explodiert die Krümmung von $g_{T}$.

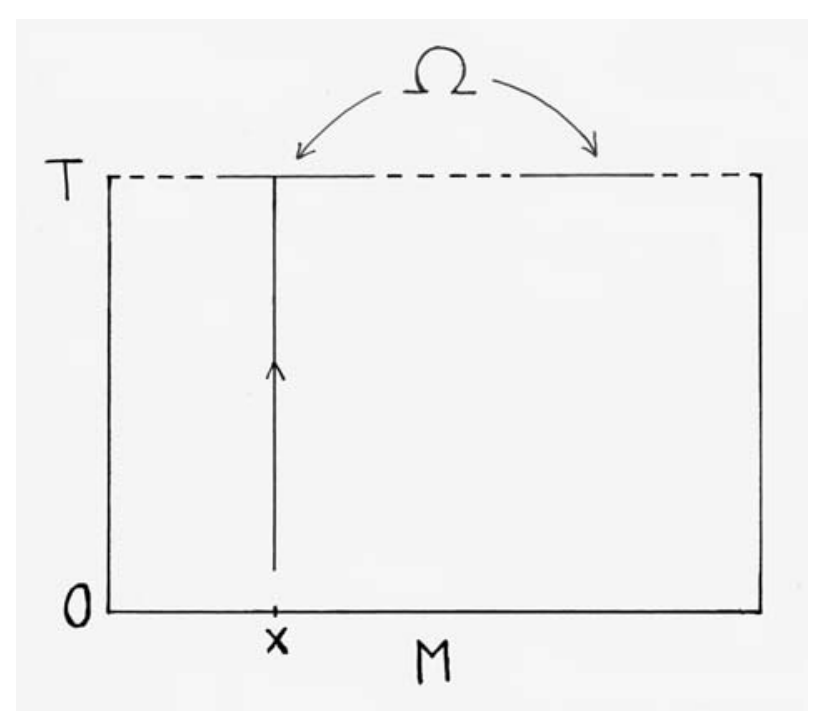

Abbildung 3. Globale Degeneration

Wie die Analyse der Urlösungen ergibt, hat jedes Ende die Gestalt eines Hornes, d. h. es läßt sich durch eine Folge immer feinerer fast-zylindrischer Röhrchen überdecken.

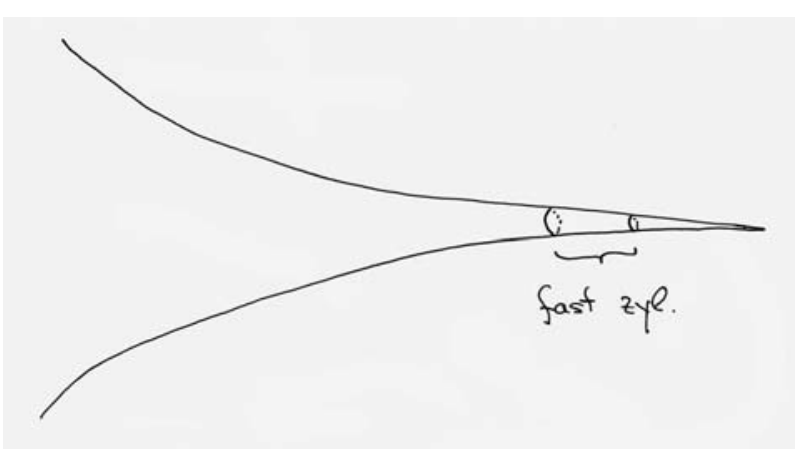

Abbildung 4. Horn

Möglicherweise ist $\Omega=\emptyset$, nämlich bei gleichmäßiger Krümmungsexplosion scal $\nearrow+\infty$, wie sie sich etwa bei positiver Ricci-Krümmung ereignet. In diesem Fall halte man den Ricci-Fluß zu einer Zeit $T-\epsilon$ kurz vor seiner Entartung an, wenn die Skalarkrümmung schon überall sehr hoch ist. Ganz $\left(M, g_{T-\epsilon}\right)$ kann dann durch metrisch gut approximierende lokale Modelle überdeckt werden, die sich außerdem gut zusammenfügen. Man sieht so, daß $M$ spezielle Topologie hat, nämlich diffeomorph ist zu einer sphärischen Raumform $S^{3} / \Gamma$ oder einer der Mannigfaltigkeiten $S^{2} \times S^{1}$ und $S^{2} \times_{\mathbb{Z}_{2}} S^{1} \cong P \mathbb{R}^{3} \sharp P \mathbb{R}^{3}$. Dieselbe Schlußfolgerung ergibt sich im Fall $\Omega \neq \emptyset$, wenn $\operatorname{scal}(\cdot, T) \simeq+\infty$, d. h. wenn die Skalarkrümmung der Limes-Metrik $g_{T}$ überall auf $\Omega$ einen gewissen Schwellenwert überschreitet.

In der generischen Situation $\Omega \neq \emptyset$ ist denkbar, daß die Menge $\Omega$ extrem kompliziert ist. Man kann nicht ausschließen, daß sie unendlich viele Komponenten hat und ihr Komplement z.B. eine Cantor-Menge ist. Weil die Regionen hoher Krümmung scal $\simeq+\infty$ geometrisch und topologisch gut verstanden sind, müssen wir jedoch nur diejenigen Komponenten $\Omega_{i}$ von $\Omega$ beachten, die auch Punkte mit nicht allzu hoher Skalarkrümmung aufweisen. Aus Volumengründen kann es nur endlich viele solcher Komponenten $\Omega_{i}$ geben und diese haben insgesamt nur endlich viele Hörner $H_{k}$. (Denn Hörner enthalten fast-zylindrische Stücke eines gewissen Mindestvolumens.)

\section{Ricci-Fluß mit Chirurgie}

Die Chirurgie zur Zeit $T$ führen wir nun simultan an den endlich vielen Hörnern $H_{k}$ durch. Wir wählen innerhalb jedes dieser Hörner einen fast-zylindrischen Abschnitt, kappen ihn entlang eines Querschnittes nahe der Mitte und ersetzen die Spitze des Hornes durch eine fast runde 3-dimensionale Hemisphäre passender Größe. Zur Chirurgiezeit $T$ hat man einen im allg. unvollständigen ,alten“ raumartigen Slice $\left(\Omega, g_{T_{-}}\right)$und einen geschlossenen „neuen“ $\left(M^{\prime}, g_{T_{+}}\right)$, die längs der gekappten Komponenten $\Omega_{i}$ übereinstimmen. (So sind die Verzweigungen in Abb. 5 gemeint.) Mit dem neuen Slice als Startbedingung setzt 


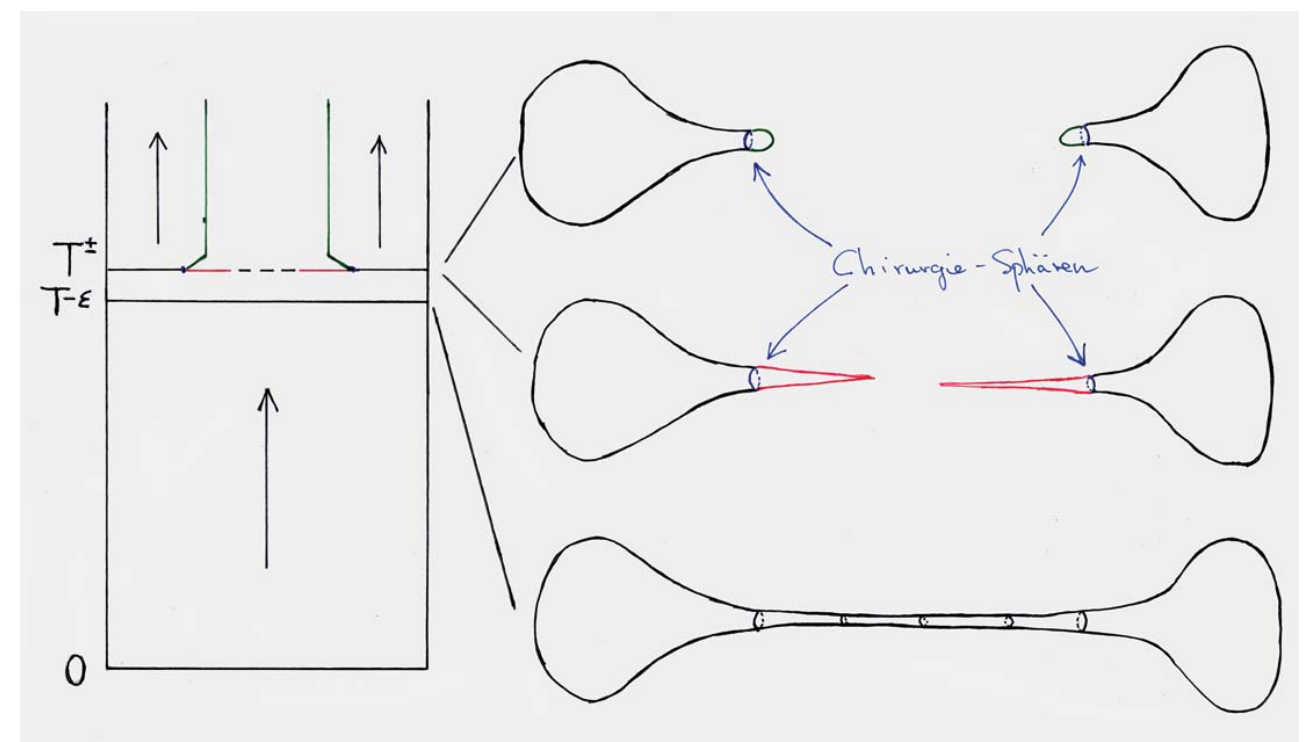

Abbildung 5. Chirurgie

man den Ricci-Fluß fort. Gibt es weitere Entartungen nach endlicher Zeit, wiederholt man die Chirurgie, und so fort.

Die dem Ricci-Fluß zugrundeliegende Mannigfaltigkeit $M$ verändert sich durch die Chirurgie. Die Regionen zwischen den Hörnern sind (bzgl. der Metriken $\left.g_{T-\epsilon}\right)$ lange dünne Hälse mit fast-runden Querschnitten langsam variierender Breite. Sie reichen möglicherweise nur an einem Ende in ein Horn hinein und können am anderen durch einen Ball oder gelochten $P \mathbb{R}^{3}$ eingefüllt sein. Der topologische Effekt der Chirurgie besteht also darin, daß $M$ entlang endlich vieler 2-Sphären zerschnitten wird, in die Randsphären Bälle eingefüllt und eventuell einige Komponenten ,,weggeworfen" werden, die zur im Fall $\Omega=\emptyset$ angegebenen Liste gehören. Letztere sind geometrisierbar!

Bei der Chirurgie ist folgende Balance einzuhalten: Die Chirurgie ist tief genug im Horn anzusetzen, damit die geometrische Kontrolle ausreicht, um sie präzise durchzuführen. Andererseits nicht zu tief, damit (beim Ersetzen der Hälfte der im Verhältnis zur Krümmungsskala langen Röhre durch die Hemisphäre) ein gewisses Quantum an Volumen verlorengeht. Dies ist wichtig, um bei Iteration des Prozesses die zeitliche Diskretheit der Chirurgien zu gewährleisten.

Damit man einen für alle Zeiten $0 \leq t<\infty$ definierten Ricci-Fluß mit Chirurgie erhält, muß man sicherstellen, daß wichtige Eigenschaften des gewöhnlichen Ricci-Flusses bewahrt bleiben, vor allem der Nichtkollaps, das Hamilton-Ivey-Pinching und die Existenz gut kontrollierter lokaler Modelle für die Regionen hoher Krümmung. Ein wesentlicher Punkt ist, die Diskretheit der auftretenden Chirurgien zu gewährleisten, d.h. daß bis zu jedem endlichen Zeit- punkt höchstens endlich viele Chirurgien stattfinden. Das Zusammenspiel der vielen beteiligten Parameter über das gesamte Zeitintervall $[0, \infty)$ hinweg zu koordinieren, gelingt Perelman durch eine sehr komplexe und fein austarierte Konstruktion [Pe2]. Eine Häufung von Chirurgien in endlicher Zeit ist dann aus Volumengründen unmöglich.

Die Konstruktion des Ricci-Flusses mit Chirurgie ist einer der fundamentalen Beiträge Perelmans. Hat man ihn zur Verfügung, so ist der größte Teil des Weges zum Beweis der Poincaré- und ThurstonVermutungen zurückgelegt.

\section{Auslöschung und Elliptisierung}

Wie wir gesehen haben, kann sich der räumliche Querschnitt im Laufe des Ricci-Flusses mit Chirurgie nicht nur ändern, sondern nach endlicher Zeit sogar ganz leer werden. Man spricht dann von Auslöschung (extinction). Genau das passiert ja im zuerst verstandenen Fall Ric $>0$ und der oben diskutierten allgemeineren Situation, wenn $\Omega=\emptyset$ bei der ersten Entartungszeit.

Auslöschung bedingt, daß nur endlich viele Chirurgien stattfinden können. Die resultierenden Komponenten verschwinden nach und nach, indem die Skalarkrümmung auf ihnen entweder gleichmäßig explodiert oder zumindest kurz vor dem Auftreten der Singularitäten überall sehr groß wird. Sie haben also spezielle Topologie, vgl. die obige Liste im Fall $\Omega=\emptyset$. Die ursprüngliche Mannigfaltigkeit $M^{3}$ selbst gehört zur Klasse $\mathcal{S}$ von 3-Mannigfaltigkeiten, deren Primfaktoren diffeomorph zu sphärischen Raumformen $S^{3} / \Gamma$ oder zu $S^{2} \times S^{1}$ sind. 


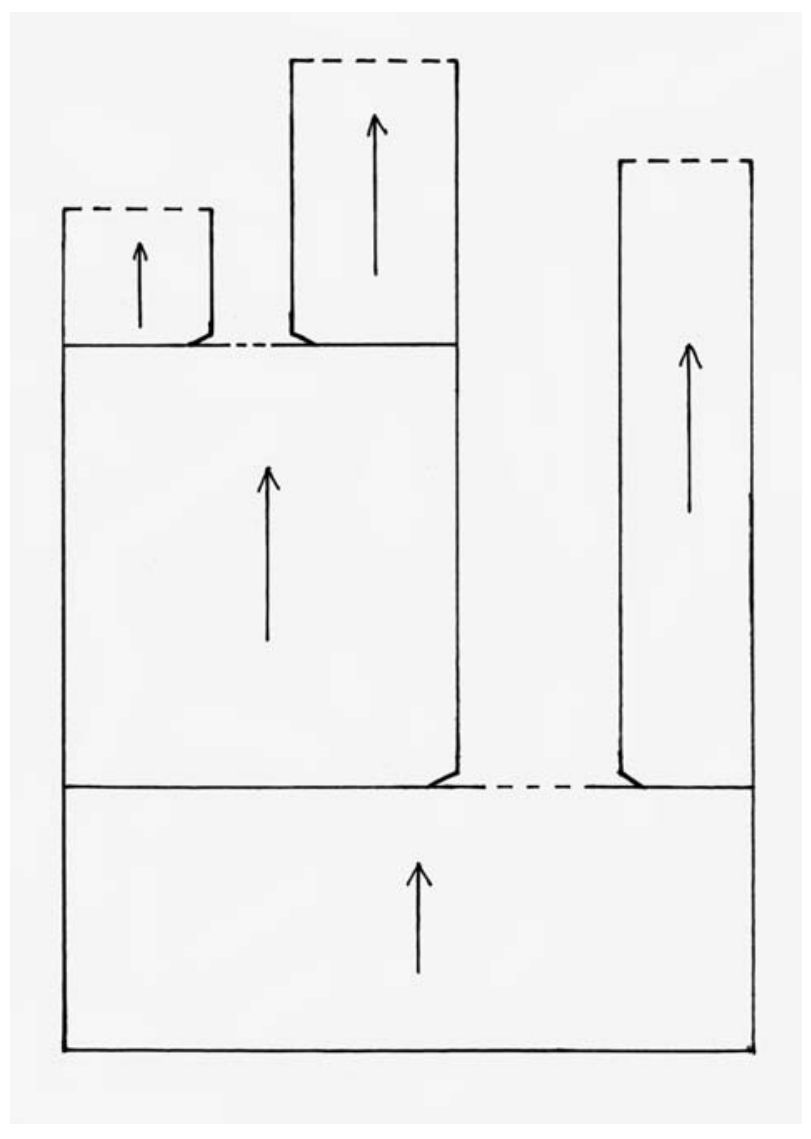

Abbildung 6. Auslöschung

Eine interessante Folgerung an dieser Stelle ist die Klassifikation orientierbarer geschlossener 3Mannigfaltigkeiten mit positiver Skalarkrümmung. Es ist leicht, auf den Mannigfaltigkeiten in $\mathcal{S}$ Metriken mit scal $>0 \mathrm{zu}$ konstruieren. Ist andererseits $g_{0}$ eine Startmetrik mit scal $>0$, so wird der RicciFluß nach endlicher Zeit ausgelöscht. Denn der ODEVergleich, der für einen gewöhnlichen Ricci-Fluß impliziert, daß min scal $(\cdot, t)$ nach endlicher Zeit explodiert, bleibt genauso auch für den Ricci-Fluß mit Chirurgie bestehen.

Die Elliptisierungs- und damit auch die PoincaréVermutung lassen sich verifizieren, ohne das asymptotische Langzeitverhalten des Ricci-Flusses zu studieren, denn auf geschlossenen 3-Mannigfaltigkeiten mit endlicher Fundamentalgruppe wird der RicciFluß mit Chirurgie für jede Startmetrik nach endlicher Zeit ausgelöscht [Pe3]. Es folgt, daß diese Mannigfaltigkeiten der Klasse $\mathcal{S}$ angehören, also wegen der Endlichkeit ihrer Fundamentalgruppe sphärische Raumformen sind. Genauer besteht der folgende $\mathrm{Zu}$ sammenhang zwischen Topologie und Auslöschung: Auslöschung ist nur auf Mannigfaltigkeiten in $\mathcal{S}$ möglich und findet umgekehrt auf diesen Mannigfaltig- keiten für jede Startmetrik statt. Ein technisch einfacherer Zugang zu diesen Resultaten mit Hilfe von Minimalflächen wurde wenig später von Colding und Minicozzi $[\mathrm{CM}]$ gegeben.

\section{Langzeitverhalten und Geometrisierung}

Für die volle Geometrisierungsvermutung untersucht man das Langzeitverhalten des Ricci-Flusses mit Chirurgie, vgl. Hamiltons Programm. Einige interessante Teilargumente wurden bereits in [H3] unter speziellen Annahmen durchgeführt.

Die Primzerlegung erfolgt nach endlicher Zeit durch einige der Chirurgien. Die 2-Sphären, entlang derer man die Mannigfaltigkeit in Primfaktoren zerschneidet, können nämlich als sich mit dem Fluß mitbewegende Minimalflächen realisiert werden. Ihr Flächeninhalt muß mit einer gewissen Rate abnehmen, vgl. [CM], weswegen sie nur für endliche Zeit überleben können. Danach sind alle Zusammenhangskomponenten des räumlichen Querschnitts prim. Andererseits sind im allg. nicht alle Chirurgien topologisch relevant, sondern je nach Startmetrik können weitere hinzukommen, die triviale $S^{3}$-Faktoren abspalten. Man kann nicht einmal ausschließen, daß insgesamt unendlich viele Chirurgien stattfinden.

Im asymptotischen Langzeitverhalten bildet sich eine Dick-Dünn-Zerlegung heraus, die in engem $\mathrm{Zu}$ sammenhang zur Torus-Zerlegung steht. Dick sind die Regionen, in denen das asymptotische Volumenwachstum von $g_{t}$ mit der Rate $\sim t^{\frac{3}{2}}$ im rein hyperbolischen Fall vergleichbar ist. Dort formieren sich endlich viele inkompressible ${ }^{3}$ hyperbolische Komponenten, von denen jede ein gewisses Quantum zum asymptotischen Volumenwachstum beiträgt. Ihre Inkompressibilität ergibt sich - über ein MinimalflächenArgument wie in [H3] - daraus, daß diese Komponenten bis zur Zeit $+\infty$ überleben. Dabei nähern sie sich immer besser vollständigen hyperbolischen Mannigfaltigkeiten endlichen Volumens an, d. h. geschlossenen oder nichtkompakten mit endlich vielen, sich exponentiell verjüngenden Enden („Spitzen“) mit Torus-Querschnitten. Die Inkompressibilität ist hier eine unverzichtbare Information, weil kompressible hyperbolische Komponenten topologisch bedeutungslos sein können. Beispielsweise gibt es auf $S^{3}$ Metriken, die außerhalb der Tubenumgebung eines Knotens eine vollständige hyperbolische Struktur beliebig gut approximieren. Solche kompressiblen hyperbolischen Komponenten lösen sich unter dem Ricci-Fluß jedoch nach endlicher Zeit wieder auf.

3 d.h. ihre Fundamentalgruppen bilden injektiv in die Fundamentalgruppen der räumlichen Slices ab. 
Die dünnen Regionen kollabieren (relativ zu einer geeigneten Normalisierung) bei unterer Schnittkrümmungsschranke. Ein solcher Kollaps findet entlang von Faserungen statt, und auch diese Regionen sind deshalb topologisch verstanden. In dieses letzte Stadium der Argumentation gehen vom Ricci-Fluß völlig unabhängige geometrische Techniken ein.

Insgesamt ergibt sich, daß die Ausgangsmannigfaltigkeit, auf welcher der Ricci-Fluß mit Chirurgie gestartet wurde, die Geometrisierungsvermutung erfüllt.

\section{Coda}

Wir konnten in dieser Note naturgemäß nur einen meist sehr groben Überblick über einige Etappen der Entwicklung versuchen. Wer die Beweise der Vermutungen genau verstehen möchte, sei neben [Pe1, Pe2, Pe3] auf die ersten detaillierten Darstellungen seiner Argumente verwiesen, nämlich das Buch [MT], in welchem die Elliptisierungs-Vermutung bewiesen wird, und für die volle Geometrisierungs-Vermutung auf $[\mathrm{KL}]$.

Durch die Arbeit von Perelman sind grundlegende und für lange Zeit offene Fragen in der 3-dimensionalen Topologie geklärt worden. Das Gebiet ist damit natürlich keinesfalls abgeschlossen, sondern hat eine neue Fundierung erfahren!

\section{Literatur}

[CM] T. Colding, W. Minicozzi, Estimates for the extinction time for the Ricci flow on certain 3-manifolds and a question of Perelman, J. Amer. Math. Soc. 18 no. 3 (2005), 561-569.
[H1] R.S. Hamilton, Three-manifolds with positive Ricci curvature, J. Diff. Geom. 17, 1982, 255-306.

[H2] R.S. Hamilton, The formation of singularities in the Ricci flow, Surveys in Differential Geometry, 2 (1995), 7-136.

[H3] R.S. Hamilton, Non-singular solutions to the Ricci flow on three-manifolds, Commun. Anal. Geom. 7 no. 4 (1999), 695-729.

[KL] B. Kleiner, J. Lott, Notes on Perelman's papers, Mathematics ArXiv math.DG/0605667, May 2006.

[MT] J.W. Morgan, G. Tian, Ricci Flow and the Poincaré Conjecture, Mathematics ArXiv math.DG/0607607, July 2006.

[Pe1] G. Perelman, The entropy formula for the Ricci flow and its geometric applications, Mathematics ArXiv math.DG/0211159, 2002.

$[\mathrm{Pe} 2] \quad$ G. Perelman, Ricci flow with surgery on threemanifolds, Mathematics ArXiv math.DG/0303109, 2003.

[Pe3] G. Perelman, Finite extinction time for the solutions to the Ricci flow on certain three-manifolds, Mathematics ArXiv math.DG/0307245, 2003.

[Poi] H. Poincaré, Analysis Situs, mit fünf Komplementen, in: CEuvres, tome VI (Géométrie), Gauthier-Villars, Paris 1953.

[Sc] P. Scott, The geometries of 3-manifolds, Bull. LMS 15 (1983), 401-487.

[T] W. Thurston, Three-Dimensional Geometry and Topology, Princeton 1997.

\section{Adresse des Autors}

Prof. Dr. Bernhard Leeb

Mathematisches Institut

Ludwig-Maximilians-Universität

Theresienstraße 39

80333 München

leeb@mathematik.uni-muenchen.de

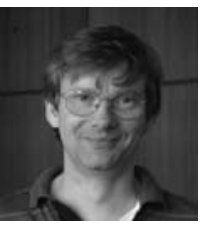

\section{mathemas ordinate www.ordinate.de}

× 0431-2374500/凶-3288812, info@ordinate.de $\rightarrow$ Software for mathematical people! Mathematica, KaleidaGraph, MathType $^{\mathrm{TM}}$, Fortran, NSBasic, Extend, Microsoft, @Risk und a.m.

mathemas ordinate, Dipl. Math.Carsten Herrmann, M. Sc. Königsbergerstr. 97, 24161 Altenholz

Fast 20 Jahre Erfahrung mit Software-Distribution! 